\author{
International Journal of Contemporary Mathematical Sciences \\ Vol. 9, 2014, no. 16, $767-776$ \\ HIKARI Ltd, www.m-hikari.com \\ http://dx.doi.org/10.12988/ijcms.2014.411118
}

\title{
Finite Difference Method of Fractional Parabolic Partial Differential Equations with Variable Coefficients
}

\author{
Ibrahim Karatay and Nurdane Kale \\ Department of Mathematics, Fatih University \\ Hadimkoy Campus, Hadimkoy road \\ 34500, Buyukcekmece, Istanbul, Turkey
}

Copyright (c) 2014 Ibrahim Karatay and Nurdane Kale. This is an open access article distributed under the Creative Commons Attribution License, which permits unrestricted use, distribution, and reproduction in any medium, provided the original work is properly cited.

\begin{abstract}
In this study, we consider the fractional parabolic partial differential equations that variable coefficients with the Caputo fractional derivative. We constructed a difference scheme based on Crank- Nicholson method. And stability of this difference scheme is proved conditionally.
\end{abstract}

Mathematics Subject Classification: 65N06, 65N12, 65M12

Keywords: Difference scheme, Stability, Caputo fractional derivative

\section{Introduction}

In this study, we propose a Crank-Nicholson type of difference scheme and we see that under some conditions the difference scheme is stable for the following time fractional partial differential problem that variable coefficients;

$$
\left\{\begin{array}{l}
\frac{\partial^{\alpha} u(x, t)}{\partial t^{\alpha}}=a(x) \frac{\partial^{2} u(x, t)}{\partial x^{2}}-b(x) \frac{\partial u(x, t)}{\partial x} \\
-c(x) u(x, t)+f(x, t),(0<x<1,0<t<1) \\
u(x, 0)=r(x), 0 \leq x \leq 1 \\
u(0, t)=0, \quad u(1, t)=0, \quad 0 \leq t \leq 1
\end{array}\right.
$$




$$
\frac{\partial^{\alpha} u(x, t)}{\partial t^{\alpha}}=\frac{1}{\Gamma(1-\alpha)} \int_{0}^{t} \frac{u_{t}(x, \tau)}{(t-\tau)^{\alpha}} d \tau, 0<\alpha<1
$$

where $\Gamma($.$) is the Gamma function.$

We can obtain some kind of subproblems from this problem which general type. One can see that if $b(x)=0$ and $c(x)=0$ then the problem transforms to time fractional diffusion problem that variable coefficient, if we choose $b(x)=0$ and $c(x)>0$ then the problem will be a time-fractional cable problem that variable coefficient when opposite chosen which means $c(x)=0$ and $b(x)>0$ the problem is called fractional advection dispersion problem that variable coefficient.

\section{Discretization of the Problem}

For some positive integers $M$ and $N$, the grid sizes in space and time for the finite difference algorithm are defined by $h=1 / M$ and $\tau=1 / N$, respectively. The grid points in the space interval $[0,1]$ are the numbers $x_{j}=$ $j h, j=0,1,2, \ldots, M$, and the grid points in the time interval $[0,1]$ are labeled $t_{k}=k \tau, k=0,1,2, \ldots, N$. The values of the functions $u$ and $f$ at the grid points are denoted $u_{j}^{k}=u\left(x_{j}, t_{k}\right)$ and $f_{j}^{k}=f\left(x_{j}, t_{k}\right)$, respectively. Let $u(x, t), u_{t}(x, t)$ and $u_{t t}(x, t)$ are continuous on $[0,1]$.

A discrete approximation to the fractional derivative $\frac{\partial^{\alpha} u(x, t)}{\partial t^{\alpha}}$ at $\left(x_{j}, t_{k+\frac{1}{2}}\right)$ can be obtained by the following approximation[7]:

$$
\begin{aligned}
\frac{\partial^{\alpha} u\left(x_{j}, t_{k+\frac{1}{2}}\right)}{\partial t^{\alpha}} & =\left[w_{1} u^{k}+\sum_{m=1}^{k-1}\left(w_{k-m+1}-w_{k-m}\right) u^{m}-w_{k} u^{0}+\sigma \frac{\left(u_{j}^{k+1}-u_{j}^{k}\right)}{2^{1-\alpha}}\right] \\
& +O\left(\tau^{2-\alpha}\right) .
\end{aligned}
$$

Where $\sigma=\frac{1}{\Gamma(2-\alpha)} \frac{1}{\tau^{\alpha}}$ and $w_{j}=\sigma\left((j+1 / 2)^{1-\alpha}-(j-1 / 2)^{1-\alpha}\right)$ In addition for $\mathrm{k}=0$ there is no these terms $w_{1} u_{k}$ and $w_{k} u_{0}$. On the other hand, we have also approximations for second and first derivative at $\left(x_{j}, t_{k+\frac{1}{2}}\right)$.

$$
\begin{gathered}
\frac{\partial^{2} u\left(x_{j}, t_{k+\frac{1}{2}}\right)}{\partial x^{2}}=\frac{1}{2}\left[\frac{u_{j+1}^{k+1}-2 u_{j}^{k+1}+u_{j-1}^{k+1}}{h^{2}}+\frac{u_{j+1}^{k}-2 u_{j}^{k}+u_{j-1}^{k}}{h^{2}}\right]+O\left(h^{2}\right) . \\
\frac{\partial u\left(x_{j}, t_{k+\frac{1}{2}}\right)}{\partial x}=\frac{1}{2}\left[\frac{u_{j+1}^{k+1}-u_{j-1}^{k+1}}{2 h}+\frac{u_{j+1}^{k}-u_{j-1}^{k}}{2 h}\right]
\end{gathered}
$$


Using these approximations (3), (4) and (5) into (1), we obtain the following difference scheme for (1) which is accurate of order $O\left(\tau^{2-\alpha}+h^{2}\right)$;

$$
\begin{aligned}
& \left\{\begin{array}{l}
{\left[w_{1} u_{j}^{k}+\sum_{m=1}^{k-1}\left(w_{k-m+1}-w_{k-m}\right) u_{j}^{m}-w_{k} u_{j}^{0}+\sigma \frac{\left(u_{j}^{k+1}-u_{j}^{k}\right)}{2^{1-\alpha}}\right]} \\
-a\left(x_{j}\right)\left[\frac{u_{j+1}^{k+1}-2 u_{j}^{k+1}+u_{j-1}^{k+1}}{2 h^{2}}+\frac{u_{j+1}^{k}-2 u_{j}^{k}+u_{j-1}^{k}}{2 h^{2}}\right]+b\left(x_{j}\right)\left(\frac{u_{j+1}^{k+1}-u_{j-1}^{k+1}}{4 h}+\frac{u_{j+1}^{k}-u_{j-1}^{k}}{4 h}\right) \\
+c\left(x_{j}\right) \frac{u_{j}^{k+1}+u_{j}^{k}}{2}=f\left(x_{j}, t_{k}+\frac{\tau}{2}\right), \\
0 \leq k \leq N-1,1 \leq j \leq M-1, \\
u_{j}^{0}=r\left(x_{j}\right), 0 \leq j \leq M, \\
u_{0}^{k}=0, \quad u_{M}^{k}=0,0 \leq k \leq N .
\end{array}\right. \\
& \left\{\begin{array}{l}
\left(-\frac{a\left(x_{j}\right)}{2 h^{2}}+\frac{b\left(x_{j}\right)}{4 h}\right) u_{j+1}^{k}+\left(-\frac{a\left(x_{j}\right)}{2 h^{2}}+\frac{b\left(x_{j}\right)}{4 h}\right) u_{j+1}^{k+1}+\left(-\frac{\sigma}{2^{1-\alpha}}+\frac{a\left(x_{j}\right)}{h^{2}}+\frac{c\left(x_{j}\right)}{2}\right) u_{j}^{k} \\
+\left(\frac{\sigma}{2^{1-\alpha}}+\frac{a\left(x_{j}\right)}{h^{2}}+\frac{c\left(x_{j}\right)}{2}\right) u_{j}^{k+1}+w_{1} u_{j}^{k}+\sum_{m=1}^{k-1}\left(w_{k-m+1}-w_{k-m}\right) u_{j}^{m}-w_{k} u_{j}^{0} \\
+\left(-\frac{a\left(x_{j}\right)}{2 h^{2}}-\frac{b\left(x_{j}\right)}{4 h}\right) u_{j-1}^{k}+\left(-\frac{a\left(x_{j}\right)}{2 h^{2}}-\frac{b\left(x_{j}\right)}{4 h}\right) u_{j-1}^{k+1} \\
=f\left(x_{j}, t_{k}+\frac{\tau}{2}\right), 0 \leq k \leq N-1,1 \leq j \leq M-1, \\
u_{j}^{0}=r\left(x_{j}\right), 0 \leq j \leq M, \\
u_{0}^{k}=0, \quad u_{M}^{k}=0,0 \leq k \leq N .
\end{array}\right.
\end{aligned}
$$

\section{Matrix Stability of the Difference Scheme}

And we consructed this difference scheme from (6)

$$
\left\{\begin{array}{l}
\left(-\frac{a\left(x_{j}\right)}{2 h^{2}}-\frac{b\left(x_{j}\right)}{4 h}\right) u_{j-1}^{k+1}+\left(\frac{\sigma}{2^{1-\alpha}}+\frac{a\left(x_{j}\right)}{h^{2}}+\frac{c\left(x_{j}\right)}{2}\right) u_{j}^{k+1}+\left(-\frac{a\left(x_{j}\right)}{2 h^{2}}+\frac{b\left(x_{j}\right)}{4 h}\right) u_{j+1}^{k+1} \\
+\left(-\frac{a\left(x_{j}\right)}{2 h^{2}}-\frac{b\left(x_{j}\right)}{4 h}\right) u_{j-1}^{k}+\left(-\frac{\sigma}{2^{1-\alpha}}+\frac{a\left(x_{j}\right)}{h^{2}}+\frac{c\left(x_{j}\right)}{2}\right) u_{j}^{k}+\left(-\frac{a\left(x_{j}\right)}{2 h^{2}}+\frac{b\left(x_{j}\right)}{4 h}\right) u_{j+1}^{k} \\
+w_{1} u_{j}^{k}+\sum_{m=1}^{k-1}\left(w_{k-m+1}-w_{k-m}\right) u_{j}^{m}-w_{k} u_{j}^{0} \\
=f\left(x_{j}, t_{k}+\frac{\tau}{2}\right), \quad 0 \leq k \leq N-1,1 \leq j \leq M-1, \\
u_{j}^{0}=r\left(x_{j}\right), 0 \leq j \leq M, \\
u_{0}^{k}=0, \quad u_{M}^{k}=0,0 \leq k \leq N .
\end{array}\right.
$$


The difference scheme above (7) can be written in matrix form,

$$
\left\{\begin{array}{l}
A U^{1}=B U^{0}+\varphi^{0}, \quad k=0 \\
A U^{k+1}=B U^{k}-w_{1} U^{k}+\sum_{m=1}^{k-1}\left(w_{k-m}-w_{k-m+1}\right) U^{m} \\
+w_{k} U^{0}+\varphi^{k}, \quad 1 \leq k \leq N-1 \\
U_{j}^{0}=r\left(x_{j}\right), 0 \leq j \leq M \\
U_{0}^{k}=0, \quad U_{M}^{k}=0,0 \leq k \leq N
\end{array}\right.
$$

where $\varphi^{k}=\left[\varphi_{1}^{k}, \varphi_{2}^{k}, \varphi_{3}^{k}, \ldots, \varphi_{M-1}^{k}\right]^{T}, \varphi_{j}^{0}=r\left(x_{j}\right), \varphi_{j}^{k}=f\left(x_{j}, t_{k+1 / 2}\right), 1 \leq k \leq N$, $1 \leq j \leq M$, and $U^{k}=\left[U_{1}^{k}, U_{2}^{k}, U_{3}^{k}, \ldots, U_{M-1}^{k}\right]^{T}$.Here, $A$ and $B$ are 3-diagonal $(M-1) \times(M-1)$ matrices of the form :

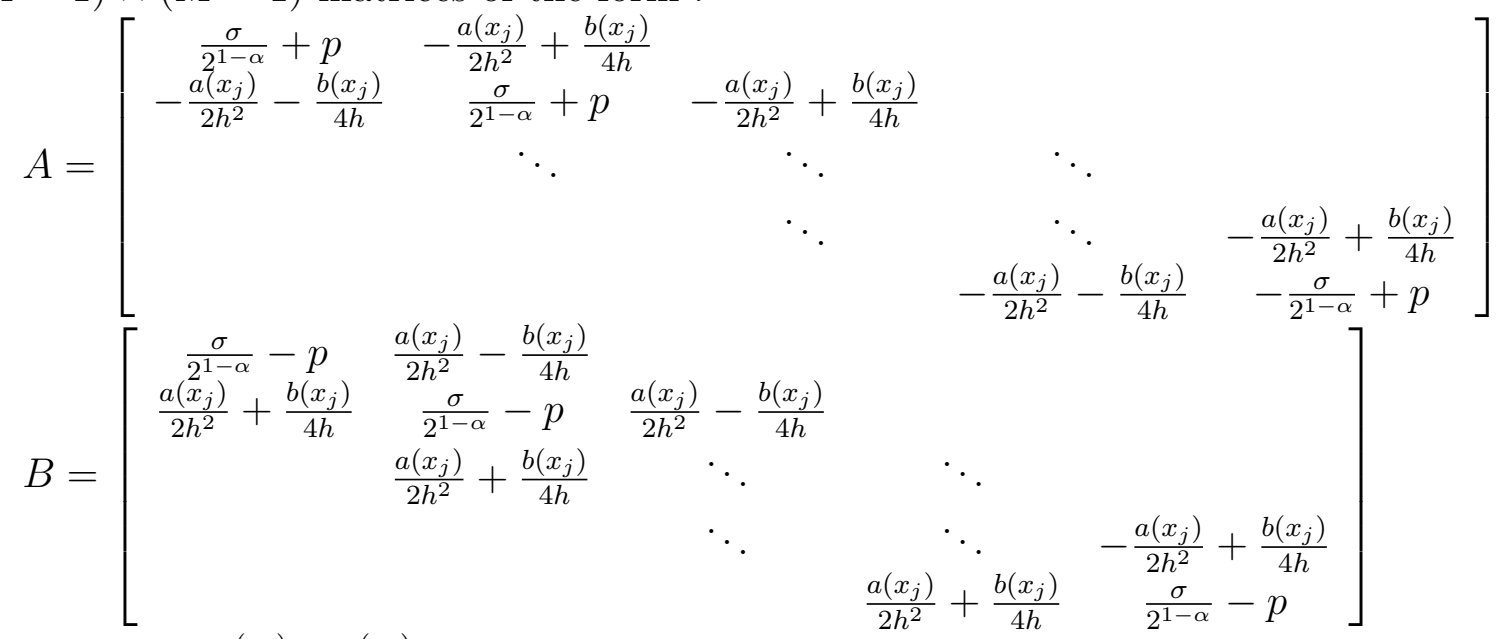

where $p=\frac{a\left(x_{j}\right)}{h^{2}}+\frac{c\left(x_{j}\right)}{2}$

We denote $\|A\|=\|A\|_{\infty}=\max _{1 \leq j \leq M-1}\left\{\sum_{m=1}^{M-1}\left|a_{j m}\right|\right\}$, where $A=\left[a_{j m}\right]_{(M-1) \times(M-1)}$.

Lemma3.1 If $\frac{\sigma}{2^{1-\alpha}}-\frac{a\left(x_{j}\right)}{h^{2}}-\frac{c\left(x_{j}\right)}{2}>0, \frac{a\left(x_{j}\right)}{b\left(x_{j}\right)}>\frac{h}{2} a\left(x_{j}\right)>0, b\left(x_{j}\right)>0$ and $c\left(x_{j}\right)>0$, then $\left\|A^{-1} B\right\| \leq 1$.

Proof. Therefore,

$$
\begin{aligned}
\left\|A^{-1} B\right\| & \leq\left\|A^{-1}\right\|\|B\| \leq \frac{1}{\min _{1 \leq j \leq M-1}\left\{\left|a_{j j}\right|-\sum_{m \neq j, m=1}^{M-1}\left|a_{j m}\right|\right\}}\|B\| \\
& \leq \frac{\left|\frac{\sigma}{2^{1-\alpha}}-\frac{a\left(x_{j}\right)}{h^{2}}-\frac{c\left(x_{j}\right)}{2}\right|+\left\{\left|\frac{a\left(x_{j}\right)}{2 h^{2}}+\frac{b\left(x_{j}\right)}{4 h}\right|+\left|\frac{a\left(x_{j}\right)}{2 h^{2}}-\frac{b\left(x_{j}\right)}{4 h}\right|\right\}}{\left|\frac{\sigma}{2^{1-\alpha}}+\frac{a\left(x_{j}\right)}{h^{2}}+\frac{c\left(x_{j}\right)}{2}\right|-\left\{\left|-\frac{a\left(x_{j}\right)}{2 h^{2}}-\frac{b\left(x_{j}\right)}{4 h}\right|+\left|-\frac{a\left(x_{j}\right)}{2 h^{2}}+\frac{b\left(x_{j}\right)}{4 h}\right|\right\}} \\
& =\frac{\frac{\sigma}{2^{1-\alpha}}-\frac{c\left(x_{j}\right)}{2}}{\frac{\sigma}{2^{1-\alpha}}+\frac{c\left(x_{j}\right)}{2}} \leq 1 .
\end{aligned}
$$

Lemma3.2 If $\frac{\sigma}{2^{1-\alpha}}-\frac{a\left(x_{j}\right)}{h^{2}}-\frac{c\left(x_{j}\right)}{2}-w_{1}>0, \frac{a\left(x_{j}\right)}{b\left(x_{j}\right)}>\frac{h}{2}, a\left(x_{j}\right)>0, b\left(x_{j}\right)>0$ and $c\left(x_{j}\right)>0$ then $\left\|A^{-1}\left(B-w_{1} I\right)\right\|+\left\|A^{-1} w_{1}\right\| \leq 1$ 
Proof.

$$
\begin{aligned}
\left\|A^{-1}\left(B-w_{1} I\right)\right\|+\left\|w_{1} A^{-1}\right\| & \leq\left\|A^{-1}\right\|\left\|B-w_{1} I\right\|+\left\|w_{1} A^{-1}\right\| \\
& \leq \frac{\frac{\sigma}{2^{1-\alpha}}-\frac{c\left(x_{j}\right)}{2}-w_{1}}{\frac{\sigma}{2^{1-\alpha}}+\frac{c\left(x_{j}\right)}{2}}+\frac{w_{1}}{\frac{\sigma}{2^{1-\alpha}}+\frac{c\left(x_{j}\right)}{2}} \\
& \leq 1
\end{aligned}
$$

Theorem3.1 The difference scheme (8) is stable.

Proof. To prove the conditional stability of (8), let $U_{j}^{k}$ and $V_{j}^{k}$ be the exact and approximate solution of (8) with initial value $U_{j}^{0}$ and $V_{j}^{0}$ respectively. We denote the corresponding error by $\varepsilon_{j}^{k}=U_{j}^{k}-V_{j}^{k}$ and $\varepsilon^{k}=\left[\varepsilon_{1}^{k}, \varepsilon_{2}^{k}, \ldots, \varepsilon_{M-1}^{k}\right]^{t}$ where $(0 \leq j \leq M, 0 \leq k \leq N)$. Then $\varepsilon^{k}$ satisfies if $k=0$

$$
A \varepsilon^{1}=B \varepsilon^{0}
$$

if $k>0$

$$
A \varepsilon^{k+1}=B \varepsilon^{k}-w_{1} \varepsilon^{k}+\sum_{m=1}^{k-1}\left(w_{k-m}-w_{k-m+1}\right) \varepsilon^{m}+w_{k} \varepsilon^{0}
$$

Let us prove $\left\|\varepsilon^{k}\right\| \leq\left\|\varepsilon^{0}\right\|, k=0,1,2, \ldots$ by induction. In fact, if $k=0$

$$
\varepsilon^{1}=A^{-1} B \varepsilon^{0}
$$

from that

$$
\left\|\varepsilon^{1}\right\|=\left\|A^{-1} B \varepsilon^{0}\right\| \leq\left\|A^{-1} B\right\|\left\|\varepsilon^{0}\right\| .
$$

Since $\left\|A^{-1} B\right\| \leq 1$, from the Lemma 1 , we have $\left\|\varepsilon^{1}\right\| \leq\left\|\varepsilon^{0}\right\|$. If $k=1$, then we have

$$
\varepsilon^{2}=A^{-1}\left(B-w_{1} I\right) \varepsilon^{1}+w_{1} A^{-1} \varepsilon^{0} .
$$

From the last equation, we obtain

$$
\begin{aligned}
\left\|\varepsilon^{2}\right\| & =\left\|A^{-1}\left(B-w_{1} I\right) \varepsilon^{1}+w_{1} A^{-1} \varepsilon^{0}\right\| \\
& \leq\left\|A^{-1}\left(B-w_{1} I\right)\right\|\left\|\varepsilon^{1}\right\|+\left\|w_{1} A^{-1}\right\|\left\|\varepsilon^{0}\right\| \\
& \leq\left\|A^{-1}\left(B-w_{1} I\right)\right\|\left\|\varepsilon^{0}\right\|+\left\|w_{1} A^{-1}\right\|\left\|\varepsilon^{0}\right\| \\
& \leq\left\{\left\|A^{-1}\left(B-w_{1} I\right)\right\|+\left\|w_{1} A^{-1}\right\|\right\}\left\|\varepsilon^{0}\right\| .
\end{aligned}
$$

If the condition above is satisfied, then $\left\|\varepsilon^{2}\right\| \leq\left\|\varepsilon^{0}\right\|$ is obtained. Now, assume 
$\left\|\varepsilon^{s}\right\| \leq\left\|\varepsilon^{0}\right\|$ for all $s \leq k$, we will prove it is also true for $s=k+1$.

$$
\begin{aligned}
\left\|\varepsilon^{k+1}\right\| & =\left\|A^{-1}\left(B \varepsilon^{k}-w_{1} \varepsilon^{k}+\sum_{m=1}^{k-1}\left(w_{k-m}-w_{k-m+1}\right) \varepsilon^{m}+w_{k} \varepsilon^{0}\right)\right\| \\
& \leq\left\|A^{-1}\left(B-w_{1} I\right)\right\|\left\|\varepsilon^{k}\right\|+\sum_{m=1}^{k-1}\left(w_{k-m}-w_{k-m+1}\right)\left\|A^{-1}\right\|\left\|\varepsilon^{m}\right\| \\
& +w_{k}\left\|A^{-1}\right\|\left\|\varepsilon^{0}\right\| \\
& \leq\left\|A^{-1}\left(B-w_{1} I\right)\right\|\left\|\varepsilon^{0}\right\|+\sum_{m=1}^{k-1}\left(w_{k-m}-w_{k-m+1}\right)\left\|A^{-1}\right\|\left\|\varepsilon^{0}\right\| \\
& +w_{k}\left\|A^{-1}\right\|\left\|\varepsilon^{0}\right\| \\
& \leq\left\|A^{-1}\left(B-w_{1} I\right)\right\|\left\|\varepsilon^{0}\right\|+\left\{\left(w_{k-1}-w_{k}\right)+\ldots+\left(w_{1}-w_{2}\right)+w_{k}\right\}\left\|A^{-1}\right\|\left\|\varepsilon^{0}\right\| \\
& \leq\left(\left\|A^{-1}\left(B-w_{1} I\right)\right\|+w_{1}\left\|A^{-1}\right\|\right)\left\|\varepsilon^{0}\right\| \\
& \leq\left\|\varepsilon^{0}\right\| .
\end{aligned}
$$

Therefore, under the condition $\frac{\sigma}{2^{1-\alpha}}-\frac{a\left(x_{j}\right)}{h^{2}}-\frac{c\left(x_{j}\right)}{2}-w_{1}>0, \frac{a\left(x_{j}\right)}{b\left(x_{j}\right)}>\frac{h}{2}, a\left(x_{j}\right)>$ $0, b\left(x_{j}\right)>0$ and $c\left(x_{j}\right)>0$, the stability inequality is obtained.

\section{Convergence Of The Difference Scheme}

Theorem4.1 The proposed scheme (8) is convergent and the following estimate holds:

$$
\left\|e^{k}\right\| \leq Z(\alpha)\left(\tau^{2-\alpha}+h^{2}\right),
$$

with the condition $\frac{\sigma}{2^{1-\alpha}}-\frac{a\left(x_{j}\right)}{h^{2}}-\frac{c\left(x_{j}\right)}{2}-w_{1}>0, \frac{a\left(x_{j}\right)}{b\left(x_{j}\right)}>\frac{h}{2}, a\left(x_{j}\right)>0, b\left(x_{j}\right)>0$ and $c\left(x_{j}\right)>0$. Here $Z(\alpha)$ does not depend on $\tau$ and $h$.

Proof. Let $R=\left[c\left(\tau^{2-\alpha}+h^{2}\right), \ldots, c\left(\tau^{2-\alpha}+h^{2}\right)\right]_{M-1}^{T}$ set $w_{0}^{-1}=1$. Since $e_{j}^{k}=$ $u_{j}^{k}-U_{j}^{k}$, notice that $e^{0}=0$. Firstly, we prove that $\left\|e^{k+1}\right\| \leq w_{n}^{-1}\|R\|$ for all $n$ by induction. We have the following error equation when $k=0$.

$$
\begin{gathered}
e^{1}=A^{-1} B e^{0}+A^{-1} R=A^{-1} R \\
\left\|e^{1}\right\|=\left\|A^{-1} R\right\| \leq\left\|A^{-1}\right\|\|R\| \leq w_{0}^{-1}\|R\| .
\end{gathered}
$$

Similarly, for $k=2$ we have the error equation

$$
e^{2}=A^{-1}\left(B-w_{1} I\right) e^{1}+w_{1} A^{-1} e^{0}+A^{-1} R
$$

If we take the norm of this equality, we obtain

$$
\begin{aligned}
\left\|e^{2}\right\| & =\left\|A^{-1}\left(B-w_{1} I\right)\right\|\left\|e^{1}\right\|+\left\|A^{-1} R\right\| \\
& \leq\left[\left\|A^{-1}\left(B-w_{1} I\right)\right\|+\left\|A^{-1} w_{1}\right\|\right] . w_{1}^{-1}\|R\| \\
& \leq w_{1}^{-1}\|R\| .
\end{aligned}
$$


Assume the inequality $\left\|e^{s}\right\| \leq w_{s-1}^{-1}\|R\|$ is true for all $s \leq k$. Now, we will prove that it is also true for $s=k+1$. We have the following error equation

$$
e^{k+1}=A^{-1}\left(B e^{k}-w_{1} e^{k}+\sum_{m=1}^{k-1}\left(w_{k-m}-w_{k-m+1}\right) e^{m}+w_{k} e^{0}+R\right)
$$

$$
\begin{aligned}
\left\|e^{k+1}\right\| & \leq\left\|A^{-1}\left(B-w_{1} I\right)\right\|\left\|e^{k}\right\| \\
& +\sum_{m=1}^{k-1}\left\|A^{-1}\left|w_{k-m}-w_{k-m+1}\right|\right\|\left\|e^{m}\right\|+\left\|A^{-1} R\right\| \\
& \leq\left\|A^{-1}\left(B-w_{1} I\right)\right\| w_{k-1}^{-1}\|R\| \\
& +\sum_{m=1}^{k-1}\left\|A^{-1}\left|w_{k-m}-w_{k-m+1}\right|\right\| w_{m-1}^{-1}\|R\|+\left\|A^{-1} w_{k}\right\| w_{k}^{-1}\|R\| \\
& \leq\left\|A^{-1}\left(B-w_{1} I\right)\right\| w_{k}^{-1}\|R\| \\
& +\sum_{m=1}^{k-1}\left\|A^{-1}\left|w_{k-m}-w_{k-m+1}\right|\right\| w_{k}^{-1}\|R\|+\left\|A^{-1} w_{k}\right\| w_{k}^{-1}\|R\| \\
& \leq\left(\left\|A^{-1}\left(B-w_{1} I\right)\right\|+\sum_{m=1}^{k-1}\left\|A^{-1}\left|w_{k-m}-w_{k-m+1}\right|\right\|+\left\|A^{-1} w_{k}\right\|\right) w_{k}^{-1}\|R\| \\
& \leq\left(\left\|A^{-1}\left(B-w_{1} I\right)\right\|+\left\|A^{-1} w_{1}\right\|\right) w_{k}^{-1}\|R\| \\
& \leq w_{k}^{-1}\|R\|
\end{aligned}
$$

Since,

$\lim _{k \rightarrow \infty} \frac{k^{-\alpha}}{\left(k+\frac{1}{2}\right)^{1-\alpha}-\left(k-\frac{1}{2}\right)^{1-\alpha}}=\frac{1}{1-\alpha}$

there exists constant $C>0$ such that

$$
\begin{aligned}
\left\|e^{k+1}\right\| & \leq w_{k}^{-1}\|R\|=k^{\alpha} k^{-\alpha} w_{k}^{-1}\|R\|=\frac{\left(k^{\alpha} \tau^{\alpha}\right) k^{-\alpha} \Gamma(2-\alpha)}{\left(k+\frac{1}{2}\right)^{1-\alpha}-\left(k-\frac{1}{2}\right)^{1-\alpha}}\|R\| \\
& \leq C \frac{\Gamma(2-\alpha)}{1-\alpha}\left(\tau^{2-\alpha}+h^{2}\right) \\
& \leq Z(\alpha)\left(\tau^{2-\alpha}+h^{2}\right),
\end{aligned}
$$

where $Z(\alpha)=C \frac{\Gamma(2-\alpha)}{1-\alpha}$. So, corresponding difference scheme is convergent under the condition. 


\section{Numerical Example}

Consider this problem,

$$
\left\{\begin{array}{l}
\frac{\partial^{\alpha} u(t, x)}{\partial t^{\alpha}}=x \frac{\partial^{2} u(t, x)}{\partial x^{2}}-\sin (x) \frac{\partial u(t, x)}{\partial x}-x^{2} u(t, x) \\
+\frac{2 t^{2-\alpha}}{\Gamma(3-\alpha)}(1-x) \sin (x)+t^{2}\left[(1-x) \sin (x)\left(x+\cos (x)+x^{2}\right)+2 x \cos (x)-\sin ^{2}(x)\right], \\
(0<x<1,0<t<1), \\
u(0, x)=0,0 \leq x \leq 1, \\
u(t, 0)=0, \quad u(t, 1)=0, \quad 0 \leq t \leq 1 .
\end{array}\right.
$$

Exact solution of this problem is $u(t, x)=t^{2}(1-x) \sin (x)$. The errors for some

$\mathrm{M}$ and $\mathrm{N}$ are given in figure 1. The errors when solving this problem are listed in the table1 for various values of time and space nodes. The errors in the table 1 are calculated by the formula $\max _{\substack{0 \leq n \leq M \\ 0 \leq k \leq N}}\left|u\left(t_{k}, x_{n}\right)-U_{n}^{k}\right|$.

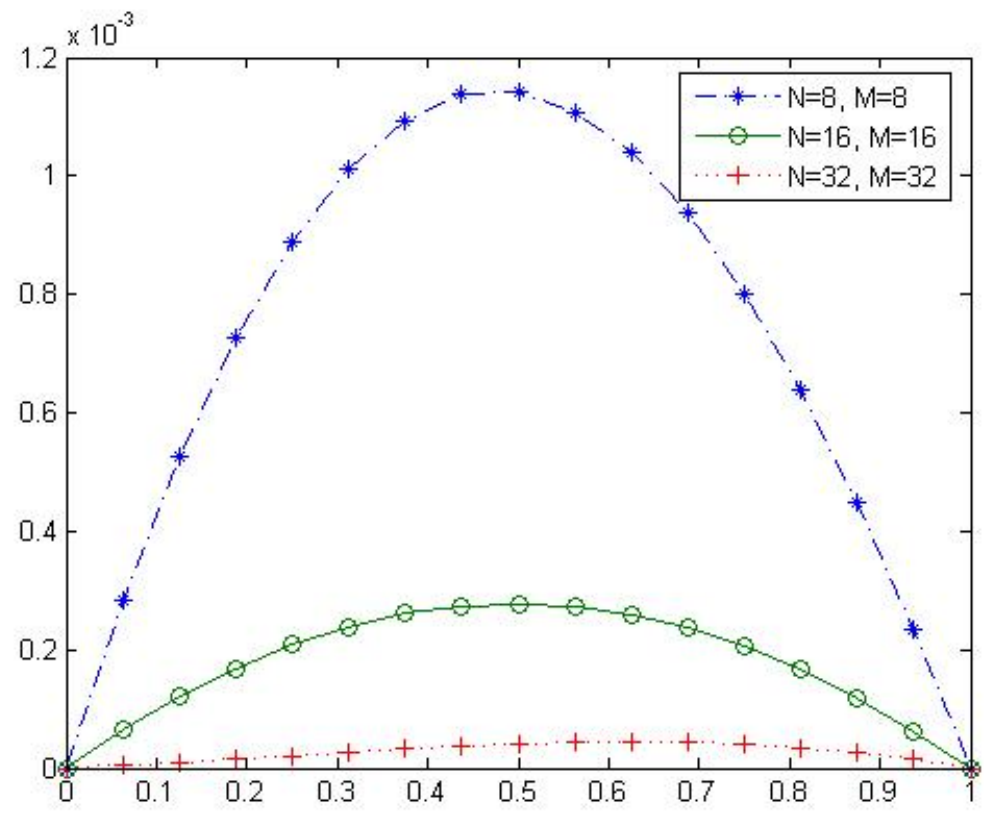

Figure 1: The errors for some values of $\mathrm{M}$ and $\mathrm{N}$ when $\mathrm{t}=1$. 
Table 1: The errors for some values of $\mathrm{M}, \mathrm{N}$ and $\alpha$

\begin{tabular}{|c|c|c|c|c|c|c|c|}
\hline & & \multicolumn{2}{|l|}{$\alpha=0.3$} & \multicolumn{2}{|l|}{$\alpha=0.5$} & \multicolumn{2}{|l|}{$\alpha=0.8$} \\
\hline$N$ & $M$ & $\operatorname{Error}(\alpha, \tau)$ & Err. rate & $\operatorname{Error}(\alpha, \tau)$ & Err. rate & $\operatorname{Error}(\alpha, \tau)$ & Err. rate \\
\hline 8 & 16 & 0.00174746 & - & 0.00154891 & - & 0.0012995 & 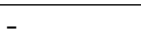 \\
\hline 16 & 16 & 0.00043279 & 4.03 & 0.00037417 & 4.1 & 0.0003855 & 3.37 \\
\hline 32 & 16 & 0.00010715 & 4.03 & 0.00009049 & 4.1 & 0.0001205 & 3.19 \\
\hline
\end{tabular}

\section{Conclusion}

In this work, $O\left(\tau^{2-\alpha}+h^{2}\right)$ order approximation for the Caputo fractional derivative based on the Crank-Nicholson difference scheme was successfully applied to solve the fractional parabolic partial differential equations with variable coefficients. It is proven that the time-fractional Crank-Nicholson difference scheme is conditionally stable. Numerical results are agreement with the theoretical results.

\section{References}

[1] A. Ashyralyev and Z. Cakir, On the numerical solution of fractional parabolic partial differential equations with the Dirichlet condition, Discrete Dynamics in Nature and Society Volume 2012 (2012), Article ID 696179, 15 pages, http://dx.doi.org/10.1155/2012/696179

[2] D. A. Benson, S. Wheatcraft, M. M. Meerschaert, Application of a fractional advection-dispersion equation, Water Resour. Res., 36 (2000), 1403-1412. http://dx.doi.org/10.1029/2000wr900031

[3] Z. Cakir, Stability of Difference Schemes for Fractional Parabolic PDE with the Dirichlet-Neumann Conditions, Volume 2012 (2012), Article ID 463746, 17 pages. http://dx.doi.org/10.1155/2012/463746

[4] C. Chen, F. Liu, I. Turner, V. Anh, A Fourier method for the fractional diffusion equation describing sub-diffusion, Journal of Computational Physics, vol. 227 (2007), 886-897.

http://dx.doi.org/10.1016/j.jcp.2007.05.012

[5] Z. Deng, V.P. Singh, L. Bengtsson, Numerical solution of fractional advection-dispersion equation, J. Hydraulic Eng. 130 (2004) 422-431. http://dx.doi.org/10.1061/(asce)0733-9429(2004)130:5(422)

[6] I. Karatay, S. R. Bayramoglu, A. Sahin, Implicit difference approximation for the time fractional heat equation with the nonlocal con- 
dition, Applied Numerical Mathematics. 61 (12) (2011), 1281-1288. http://dx.doi.org/10.1016/j.apnum.2011.08.007

[7] I. Karatay, N. Kale, S. R. Bayramoğlu, A new difference scheme for time fractional heat equations based on the Crank-Nicholson method, 16 (4)(2013), 892-910. http://dx.doi.org/10.2478/s13540-013-0055-2

[8] I. Karatay, S. R. Bayramoglu. A Characteristic Difference Scheme for Time-Fractional Heat Equations Based on the Crank-Nicholson Difference Schemes, Abstract and Applied Analysis, (2012), 11 pages, Article ID 548292. http://dx.doi.org/10.1155/2012/548292

[9] X. Li, Xianhong Han, Xuanping Wang, Numerical modeling of viscoelastic flows using equal low-order finite elements, Comput. Methods Appl. Mech. Engrg., 199 (2010), 570-581. http://dx.doi.org/10.1016/j.cma.2009.10.010

[10] X. Li, M. Xu, X. Jiang, Homotopy perturbation method to time-fractional diffusion equation with a moving boundary, Appl. Math. Comput., 208 (2009), 434-439. http://dx.doi.org/10.1016/j.amc.2008.12.023

[11] V. E. Lynch, B. A. Carreras, D. del-Castillo-Negrete, K. M. FerreiraMejias, H. R. Hicks, Numerical methods for the solution of partial differential equations of fractional order, J. Comput. Phys. 192 (2003), 406-421. http://dx.doi.org/10.1016/j.jcp.2003.07.008

[12] J. A. T. Machado, Discrete-time fractional-order controllers, Fractional Calculus Applied Analysis, 4 (1) (2001), 47-66.

[13] I. Podlubny, Fractional Differential Equations, Academic Press, New York, 1999.

[14] M. Raberto, E. Scalas, F. Mainardi, Waiting-times returns in high frequency financial data: an empirical study,Physica A, 314 (2002), 749-755. http://dx.doi.org/10.1016/s0378-4371(02)01048-8

[15] L. Su, W. Wang, Z. Yang, Finite difference approximations for the fractional advection-diffusion equation, Physics Letters A, 373(2009), 44054408. http://dx.doi.org/10.1016/j.physleta.2009.10.004

[16] C. Tadjeran, M. M. Meerschaert, H. -P. Scheffler, A Secondorder Accurate Numerical Approximation for the Fractional Diffusion Equation, Journal of Computational Physics, 213 (2006), 205-213. http://dx.doi.org/10.1016/j.jcp.2005.08.008

Received: November 21, 2014; Published: December 30, 2014 